\title{
Effect of iron deficiency anemia on lipid peroxidation and antioxidant systems
}

\author{
N. L. Madhikarmi ${ }^{1}$, K. R. S. Murthy ${ }^{2}$ \\ ${ }^{1}$ ICCR Research Scholar, ${ }^{2}$ Associate Professor, Department of Biochemistry, Central College Campus, Bangalore University, \\ Bangalore-560 001, India
}

\begin{abstract}
Iron deficiency is one of the most widespread nutritional deficiencies in the world. Globally more than two billion people are suffering from iron deficiency anemia. The present study was designed to investigate the influence of a large number of factors known to be associated with oxidative stress.
\end{abstract}

The case-control study determines the lipid peroxidation and antioxidants status in forty Iron deficiency anemia and forty healthy volunteers with their informed consent. All the parameters were assayed by spectrophotometric methods.

Blood hemoglobin and plasma iron were decreased whereas total iron binding capacity was increased significantly in Iron deficiency anemia. The lipid peroxidation parameters like malondialdehyde, lipid hydroperoxide were significantly increased in Iron deficiency anemia. Both enzymatic; glutathione peroxidase, superoxide dismutase and catalase and non-enzymatic antioxidants; vitamin $\mathrm{C}, \mathrm{E}$ and reduced glutathione were significantly decreased in Iron deficiency anemia case as compared to their healthy counterparts.

Our findings suggest, increased lipid peroxidation products and reduced antioxidants system boost the oxidative stress state, hence deteriorating the condition of Iron deficiency anemia patients.

Keywords: CAT, GPx, Iron deficiency Anemia, Oxidative stress.

\section{Introduction}

Iron deficiency anemia (IDA) is one of the most widespread nutritional deficiencies and globally more than two billion people are suffering from this condition. Oxidative stress (OS) has long been demonstrated, but the factors influencing their Correspondence: N.L. Madhikarmi

E-mail: nirjala4@gmail.com oxidative status have not been characterized extensively. Oxidative stress is associated with increased morbidity particularly in IDA. ${ }^{1,2}$

World Health Organization (WHO) definitions for anemia differs by age, gender and pregnancy status as follows: for children 6months to 5years of age anemia is defined as a hemoglobin $(\mathrm{Hb})$ level < $11 \mathrm{gm} / \mathrm{dl}$, children $5-11$ years of age $\mathrm{Hb}<11.5 \mathrm{~g} / \mathrm{dl}$, adult male $\mathrm{Hb}$ level $<13 \mathrm{~g} / \mathrm{dl}$, non-pregnant females 


\section{N. L. Madhikarmi et al, Effect of iron deficiency anemia on lipid peroxidation and antioxidant systems}

$\mathrm{Hb}<12 \mathrm{~g} / \mathrm{dl}$. Severe anemia is defined as $\mathrm{Hb}<7.0 \mathrm{~g} /$ dl. Iron deficiency anemia was defined as the presence of anemia and serum ferritin $<12 \mathrm{ng} / \mathrm{ml}$ in children younger than 15 years and female all ages, and serum ferritin $<18 \mathrm{ng} / \mathrm{ml}$ in males age 15 years and older. Over the past decade, anemia has emerged as a risk factor that is associated with a variety of adverse outcomes in humans, including hospitalization, disability, and mortality. ${ }^{3-5}$

OS plays an important role in the pathogenesis of IDA by prooxidant and antioxidant balance in favor of the prooxidant, leading to potential damage. Free radicals are atom (e.g. oxygen, nitrogen) with at least one unpaired electron in the outermost shell, and have independent existing capability. Numerous free radicals formed within the body, (oxygen centered) most commonly the superoxide anion $\left(\mathrm{O}_{2}{ }^{--}\right)$, the hydroxyl radical $\left(\mathrm{OH}^{\mathrm{i} \%}\right)$, singlet oxygen and hydrogen peroxide $\left(\mathrm{H}_{2} \mathrm{O}_{2}\right)$ are studied. ${ }^{6-10}$ Defects in enzymes critical to the OS response have been implicated in human diseases ranging from mild chronic hemolysis to severe acute hemolysis. Because of concomitant reduction in the normal RBC (red blood cell) life span, these disease states are characterized by a compensatory increase in erythropoesis. ${ }^{11}$ The deleterious effects of OS, such as damage to cellular proteins, DNA and lipids are well characterized. However, factors that regulate the OS response and the life span of erythrocytes are less clear. ${ }^{10-13}$

The first stage of ID involves the depletion of tissue iron stores. In the absence of inflammation, the level of serum ferritin has been shown to be a sensitive and reliable biomarker of the body iron store. This stage is characterized by serum ferritin levels that are below the normal range $(<12 \mathrm{ng} / \mathrm{ml})$, without functional changes. The second stage begins when the negative balance persists. Laboratory findings in this stage include a reduction in serum iron and transferring saturation (TS) levels $(<15 \%)$, and an increase in the free erythrocyte protoporphyrin level and the total iron binding capacity. At this stage, work capacity can be reduced. The third stage, IDA occurs when iron stores are almost empty, leading to reduced erythropoiesis and $\mathrm{RBC}$, which results in the development of anemia ( Hb level $<12 \mathrm{~g} / \mathrm{dl}$ ). Anemia is associated with a low work capacity, a poor pregnancy outcome, as well as lasting effects on learning and cognitive function, attention, behavior, health and growth. ${ }^{2,2,13}$

\section{Materials and Methods}

Study design and subject recruitment- The study group included iron deficient anemic (IDA) case (40) and healthy controls (40) with their informed consent. Controls were selected on the basis of their non smoking, non alcoholic habit and were nor without any diseases or sickness. Height and weight was measured for respective body mass index (BMI) and surface area (SA). The study group subjects age ranged from 15-45 years. Description criteria for IDA are hemoglobin concentration $<11.5 \mathrm{~g} / \mathrm{dl}$ in women and $<13 \mathrm{~g} / \mathrm{dl}$ in men, plasma iron concentration $<45 \mathrm{~g} / \mathrm{dl}$ and total iron binding capacity $>60 \mathrm{imol} / 1$.

Blood was collected in tubes containing ethylenediamine tetraacetic acid (EDTA), centrifuged at $4000 \mathrm{rpm}$ for $10 \mathrm{~min}$ and the plasma was carefully separated. Erythrocyte pellet was 
Journal of College of Medical Sciences-Nepal, 2011, Vol-7, No-4

washed thrice with chilled physiological saline and RBC was lysed. All readings were measured by Shimadzu spectrophotometer. $\mathrm{Hb}$ was determined by the Beacon Diagnostics and iron and TIBC (total iron binding capacity) was assayed by Coral Clinical Systems kit.

Lipid peroxidation products was estimated using thiobarbituric acid reactive substances (TBARS) methods, which measures pink colored the MDA reactive products (Buege and Aust method) at $535 \mathrm{~nm} .{ }^{14}$ The total hydroperoxides (LOOH) in plasma was determined by the method of Jiang et al, $1992 .{ }^{15} \mathrm{LOOH}$ were detected by their ability to oxidize ferrous ion under acidic condition in the presence of xylenol orange resulting in the formation of a chromophore at $560 \mathrm{~nm}$.

Superoxide dismutase (SOD) activity in hemolysed RBC was determined by Kakkar method ${ }^{16}$ based on the $50 \%$ inhibition of the formation of nicotinamide adenine dinucleotide (NADH)phenazine methosulfate-nitroblue tetrazolium formazan at $560 \mathrm{~nm}$. Catalase (CAT) activity in hemolysed RBC was assessed by Sinha method. ${ }^{17}$ Briefly, $\mathrm{H}_{2} \mathrm{O}_{2}(0.2 \mathrm{M})$ was used as a substrate and the decrease in $\mathrm{H}_{2} \mathrm{O}_{2}$ concentration at $22^{\circ} \mathrm{C}$ in phosphate buffer $(0.05 \mathrm{M}, \mathrm{pH} 7.0)$ was read at $240 \mathrm{~nm}$. Glutathione peroxidase (GPx) activity was analyzed by the method of Rotruck. ${ }^{18}$ Reduced Glutathione (GSH) in whole blood was measured using the method of Beutler method. ${ }^{19}$

Vitamin C was estimated by Natelson ${ }^{20} 2$, 4dinitrophenyl hydrazine (DNPH) method, Vitamin E was measured by Baker method ${ }^{21}$ Total antioxidant activity (TAA) was performed according to Benzie \& Strain. ${ }^{22}$ SPSS (Statistical package for social sciences) for windows version 13.0 (SPSS, Chicago, Il, USA) was used for statistics. The results were reported as means \pm standard deviation (SD). Differences between the groups were determined by means Student's t-test. Pearson's correlation (r) was performed on paired data obtained by the individual IDA cases. Significance level was set at $\mathrm{p}<0.05$.

\section{Results and Discussion}

Our study group included twenty male and twenty female both in case and control group. The age ranged from 15-45 years both in case and control groups. BMI of both male and female were within the normal range both in case and control group. Likewise, Surface area (SA) was also within the normal range in both groups. None of the participants told that they remember iron deficiency anemia in the family. About $30 \%$ of male case and $39.55 \%$ of female IDA cases were vegetarian whereas $11.27 \%$ male case $18.71 \%$ of female IDA cases were non-vegetarian. Both IDA case and control neither were under any medication nor were on drugs supplementation (Table 1).

$\mathrm{Hb}$, iron concentration both in male and female IDA cases was below the normal range but TIBC was found to be increased. The age and gender matched male and female control groups had hemoglobin, iron and TIBC within normal range. The antioxidant enzymes catalase, superoxide dismutase and glutathione peroxidase also altered when compared with control groups. The nonenzymatic antioxidants vitamin $\mathrm{C}, \mathrm{E}, \mathrm{GSH}$ and 
N. L. Madhikarmi et al, Effect of iron deficiency anemia on lipid peroxidation and antioxidant systems

TAA lowered than that of the controls. An elevation in the lipid peroxidation was determined with diminished antioxidant activities which were significant statistically (Table 2).

TBARS was significantly correlated with respect to vitamin $\mathrm{C}$ and TAA according to Pearson's correlation. Similarly, GPx was also positively correlated with the TBARS and vitamin C (Table: 3). On plotting the graph, $\mathrm{Hb}$ level in male and female IDA against percentage of total cases, the lowest $\mathrm{Hb}$ range in male and female IDA case were $7.0-7.9 \mathrm{~g} / \mathrm{dl}$ and $5.0-6.9 \mathrm{~g} / \mathrm{dl}$ respectively. The frequency of male and female patients was found to be in $\mathrm{Hb}$ range $11.0-11.9$ and $10.0-10.9 \mathrm{~g} / \mathrm{dl}$ respectively (figure1).

TBARS was increased in both sex but the increase was significant only in males at $p<0.05$. On statistical analysis we found a significant relation of diminished hemoglobin, iron and TIBC in either sex as compared with their healthy counterparts. On the other hand, antioxidant enzymes analyzed showed increased level of catalase, but decreased SOD and GPx in RBC both in male and female cases as compared with respective counterparts. Glutathione was also increased in both sex statistically.

If not treated, iron deficiency anemia may cause stunted growth, impaired mental development, poor school performance, reduced productivity, increased morbidity and mortality, and lower selfesteemed. Iron is essential for all eukaryotes and most prokaryotes, where it is used in the synthesis of heme, iron-sulfur (FeS), and other cofactors. Fe$\mathrm{S}$ proteins are involved in catalysis, redox reactions, respiration, DNA replication, and transcription. Iron homeostasis is tightly regulated to avoid iron toxicity or iron deficiency in normal condition. In human systemic iron metabolism, iron uptake, trafficking, export and fortification are highly regulated. ${ }^{4,23-25}$

RBC homeostasis is an excellent example of redox balance: erythroid progenitors accumulate hemoglobin during development, and erythrocytes continuously transport large amounts of oxygen over the course of their approximately 120 day lifespan resulting in a high level of OS. ${ }^{11,24,26} \mathrm{RBCs}$ have evolved to have an extensive array of antioxidants to counter the level of stress, including membrane OS, cellular antioxidants such as catalase and SOD, and enzymes that continuously produce reducing agents through the glutathione systems. ${ }^{27}$ Mammalian erythrocytes utilize different antioxidant defenses that work in tandem to cope with free radical generation. ${ }^{26} \mathrm{SOD}$ catalyzes the dismutation of $\mathrm{O}_{2}^{--}$to $\mathrm{H}_{2} \mathrm{O}_{2}$. Catalase and $\mathrm{GSH}$ function to catalyze the degradation of $\mathrm{H}_{2} \mathrm{O}_{2}$ to $\mathrm{H}_{2} \mathrm{O}$ and $\mathrm{O}_{2}$. Albumin is an important extracellular antioxidant by virtue of its thiol groups, which protects vital targets such as erythrocytes from copper ion-induced Fenton reaction and inhibits iron-dependent free radical production. Lipid peroxidation of the erythrocytes causes membrane injury, osmotic fragility and destruction of the cell. ${ }^{6}$

The erythrocytes membrane is rich in polyunsaturated fatty acids, a primary target for reactions involving free radicals, ${ }^{27}$ and is very susceptible to lipid peroxidation. The significant increase of TBARS generation shown in this study reflects increased lipid peroxidation and points to 
increased levels of free radicals in plasma and RBC of IDA patients. ${ }^{1,25,28}$ Previous studies also reported increased amount of lipid peroxidation by products in the erythrocytes and plasma. ${ }^{29,30,31}$

Garg et al $2005^{33}$ supplemented vitamin A and iron (ferrous sulphate, $6 \mathrm{mg} / \mathrm{kg} / \mathrm{d}$ ) in preschool children (1-4years age) with microcytic anemia (Hb less than $10 \mathrm{~g} / \mathrm{dl}$ ) found increased $\mathrm{Hb}$ and serum iron. A study carried out by Gadjeva et al $2005^{27}$ reported higher lipid peroxidation products in plasma of IDA. The enzymatic antioxidants SOD, GPx and catalase were diminished in RBC lysates. Even after iron supplementation, enzymatic antioxidants were higher for patients with iron deficiency anemia as before therapy. Some investigators speculated that the iron- sufficient rats were better able to eliminate oxygen radicals, whereas iron deficiency increased susceptibility to oxidative stress. The increase of lipid peroxidation in pernicious anemia and hemolytic anemia was comparatively less than that of iron deficient patients. ${ }^{11,34}$

Decreased GPx activity in patients with iron deficiency anemia, and suggested that iron could be of crucial importance for erythrocyte GPx activity. In the present study, CAT activity was significantly higher in patients with iron deficiency anemia compared to the control group. In addition we observed a direct positive correlation between plasma TBARS and erythrocyte GPx levels in patients with IDA. This relationship shows the continuous oxidative stress present in IDA patients. Their result showed that after therapy with iron in IDA patients, only the level of plasma ceruloplasmin was normalized. TBARS remained high as well as antioxidant enzymes. They reported that repletion of IDA with iron promotes oxidative stress. The causative factor for the generation of hydroxyl radical is because of excess iron. ${ }^{35}$

There was an increased oxidative stress and imbalance in the antioxidant defense in patients with IDA. Patients with IDA actually are in a risk of oxidative injury after iron treatment. A diet containing high amount of vitamin $\mathrm{A}, \mathrm{C}$ and $\mathrm{E}$ is recommended during the course of iron therapy. In recent years, researchers have focused on the pathological role of free radicals in a variety of diseases, among which the most common is anemia. High levels of oxidative result in the peroxidation of cell membrane lipids by generating lipid peroxides that can decompose into multiple mutagenic carbonyl products. TBARS and LOOH are considered to be mutagenic and carcinogenic. They can also modulate the expression of genes that are related to tumor promotion. Antioxidants have been shown to inhibit both the initiation and promotion in carcinogenesis as well as counteract cell immortalization and transformation. In the present study, significantly increased TBARS and LOOH were observed in patients with IDA. This could be attributed to the increased formation or inadequate clearance of free radicals by the cellular antioxidants. The present observations are in agreement with other reports on IDA. ${ }^{8,27}$

Cellular non-enzymatic antioxidants are also known as free radical scavengers that protect a cell against toxic free radicals. Reduced GSH is the chief constituent of the thiol pool and a vital intracellular scavenger of free radicals. Therefore, 
N. L. Madhikarmi et al, Effect of iron deficiency anemia on lipid peroxidation and antioxidant systems decreased GSH levels may reflect a depletion of non-enzymatic antioxidant reserves. On the other hand, GSH plays a prominent role in the antioxidant defense system, and in the reactions of catalysis, regulation, electron transportation and in preserving the correct structure of proteins. Decreased levels of total GSH have been reported in various pathologies, including anemia., 4, 27

Lipid peroxidation is a unique mode of oxidative injury which is triggered and promoted by different radical and non-radical members of the reactive oxygen species (ROS) family or by the catalytic decomposition of preformed lipid hydroperoxides in tissues by several agents. Antioxidant enzymes such as SOD, CAT and GPx, are important for cellular protection due to their ability to detoxify free radicals, such as ROS. ${ }^{31}$ Oxidative stress, followed by lipid peroxidation, can be assessed by measuring the lipid peroxidation products and antioxidant activities. Under conditions of oxidative stress, lipid peroxidation products, such as MDA, are increased, and the levels of the antioxidants SOD and GPX are reduced. The human body conserves antioxidants in order to neutralize or limit the oxidative damage caused by free radicals. ${ }^{9,27}$ Glutathione is a crucial component of the antioxidant defense mechanism and it functions as a direct reactive free radical scavenger. Iron is necessary for maintaining normal structure and function of virtually all mammalian cells and is also involved in the immune and non-immune host defense. ${ }^{4,31}$ Iron deficiency causes deficiencies of many iron-dependent enzymes and heme containing proteins, and is known to produce many systemic abnormalities. ${ }^{27,34}$

OS that occurs in the cells, as a consequence of an inequity between the prooxidant, antioxidant systems, causes injury to biomolecules such as nucleic acids, proteins, structural carbohydrates, and lipids. In addition, a variety of lipid byproducts are produced as a consequence of lipid peroxidation some of which can exert adverse and/ or beneficial biological effects. It can also disturb the assembly of the membrane, causing changes in fluidity and permeability, alterations of ion transport and inhibition of metabolic processes. Injure to mitochondria induced by lipid peroxidation can direct to further ROS generation. ${ }^{26,27,33}$

Iron deficiency (ID) is the most widespread nutritional problem, and affects over two billion people. It is a particularly common disorder among infants, preschool-aged children, young women and older people, but it can occur at all ages and in any region. A high demand for iron during rapid growth, pregnancy and lactation, accompanied by dietary deficiencies and menstrual blood loss, are the most common causes of iron deficiency in children, young women and elderly population. ${ }^{28-30}$ It affects one's development, growth and resistance to infections, and is associated with mortality among children younger than two years old. ${ }^{33}$ The significant goal of this type of interventions is to maintain maternal and neonatal health. The results of this study indicate that iron supplementation is required for the target group..$^{34,35}$ 
Journal of College of Medical Sciences-Nepal, 2011, Vol-7, No-4

Table 1: Demographic data of patients with Iron Deficiency Anemia.

\begin{tabular}{lcccc}
\hline Characteristics & \multicolumn{2}{c}{ IDA-Case } & Control & \\
& male & female & male & female \\
\hline Number & 20 & 20 & 20 & 20 \\
Mean age (yr) & $33.35 \pm 9.94$ & $26.65 \pm 6.79$ & $33.14 \pm 8.98$ & $35.15 \pm 7.36$ \\
BMI $\left(\mathrm{kg} / \mathrm{m}^{2}\right)$ & $22.14 \pm 7.47$ & $23.64 \pm 8.16$ & $21.23 \pm 2.26$ & $20.54 \pm 2.29$ \\
SA $\left(\mathrm{m}^{2}\right)$ & $1.85 \pm 0.58$ & $1.78 \pm 0.21$ & $1.68 \pm .011$ & $1.72 \pm 0.38$ \\
Family history (IDA) & $\mathrm{No}$ & $\mathrm{No}$ & No & No \\
Fruits \&vegetables intake & daily & daily & daily & daily \\
Diet- veg (\%) & 30.45 & 39.55 & 12.15 & 28.79 \\
\multicolumn{1}{c}{$\quad$ non-veg (\%) } & 11.27 & 18.71 & 76.41 & 35.47 \\
Drug supplementation & No & No & No & No \\
\hline
\end{tabular}

Note: Age-years, BMI-Body Mass Index-kg/m², SA-surface area $\left(\mathrm{m}^{2}\right)$

Table 2: Biochemical findings of Iron Deficient Anemic patients.

\begin{tabular}{|c|c|c|c|c|}
\hline \multirow[t]{2}{*}{ Parameters } & \multicolumn{2}{|r|}{ IDA-Case } & \multicolumn{2}{|c|}{ Control } \\
\hline & Male $(n=20)$ & female $(n=20)$ & male $(n=20)$ & female $(n=20)$ \\
\hline \multicolumn{5}{|l|}{ Iron status } \\
\hline $\mathrm{Hb}(\mathrm{g} / \mathrm{dl})$ & $11.60 \pm 1.88 *$ & $9.63 \pm 2.18^{*}$ & $16.07 \pm 1.85$ & $13.46 \pm 1.39$ \\
\hline Iron $(\mathrm{g} / \mathrm{dl})$ & $53.12 \pm 3.99 * *$ & $27.22 \pm 0.23 * *$ & $157.20 \pm 14.16$ & $83.22 \pm 10.72$ \\
\hline TIBC (ìmol/1) & $87.27 \pm 5.17 *$ & $93.23 \pm 8.87^{*}$ & $50.27 \pm 3.65$ & $65.83 \pm 5.77$ \\
\hline \multicolumn{5}{|c|}{ Oxidative stress marker } \\
\hline TBARS-p (nmol/ml) & $1.88 \pm 1.45^{*}$ & $2.75 \pm 1.81$ & $5.15 \pm 2.85$ & $3.56 \pm 2.61$ \\
\hline LPHO-p (nmol/ml) & $3.53 \pm 2.29^{*}$ & $3.52 \pm 2.05^{*}$ & $0.54 \pm 0.25$ & $0.53 \pm 0.24$ \\
\hline \multicolumn{5}{|c|}{ Plasma antioxidant system } \\
\hline Vit C (mg/dl) & $0.58 \pm 0.17 *$ & $0.66 \pm 0.13 * *$ & $0.69 \pm 0.46$ & $0.85 \pm 0.15$ \\
\hline Vit E (mg/dl) & $0.69 \pm 0.07$ & $0.65 \pm 0.09$ & $1.84 \pm 0.62$ & $1.99 \pm 0.30$ \\
\hline TAA (ìmol/l) & $636.31 \pm 25.87 * *$ & $584.92 \pm 12.22 * *$ & $844.60 \pm 39.83$ & $783.78 \pm 58.88$ \\
\hline \multicolumn{5}{|c|}{ Erythrocyte antioxidant system } \\
\hline SOD (U/mgHb) & $20.20 \pm 0.67 * *$ & $23.03 \pm 0.81 *$ & $21.90 \pm 6.55$ & $25.44 \pm 11.27$ \\
\hline GPx (U/mgHb) & $3.85 \pm 1.27$ & $4.89 \pm 1.71$ & $4.16 \pm 1.39$ & $4.91 \pm 2.01$ \\
\hline CAT (U/gHb) & $750.37 \pm 38.46^{*}$ & $708.76 \pm 31.10$ & $413.53 \pm 83.18$ & $653.56 \pm 42.95$ \\
\hline GSH (mg/dl) & $8.93 \pm 10.94 * *$ & $18.08 \pm 13.19 * *$ & $50.05 \pm 12.95$ & $48.18 \pm 12.04$ \\
\hline
\end{tabular}

$* * \mathrm{p}<0.005,{ }^{*} \mathrm{p}<0.05$, 
N. L. Madhikarmi et al, Effect of iron deficiency anemia on lipid peroxidation and antioxidant systems

\section{Table 3: Correlation of IDA cases according to Pearson's method.}

\begin{tabular}{rlr}
\hline Parameter & Pearson's correlation & Significance \\
TBARS-p & & \\
Vit C & 0.6 & 0.000 \\
TAA & 0.517 & 0.007
\end{tabular}

GPx

$\begin{array}{lll}\text { TBARS-p } & 0.346 & 0.029\end{array}$

Vit C $\quad 0.333 \quad 0.036$

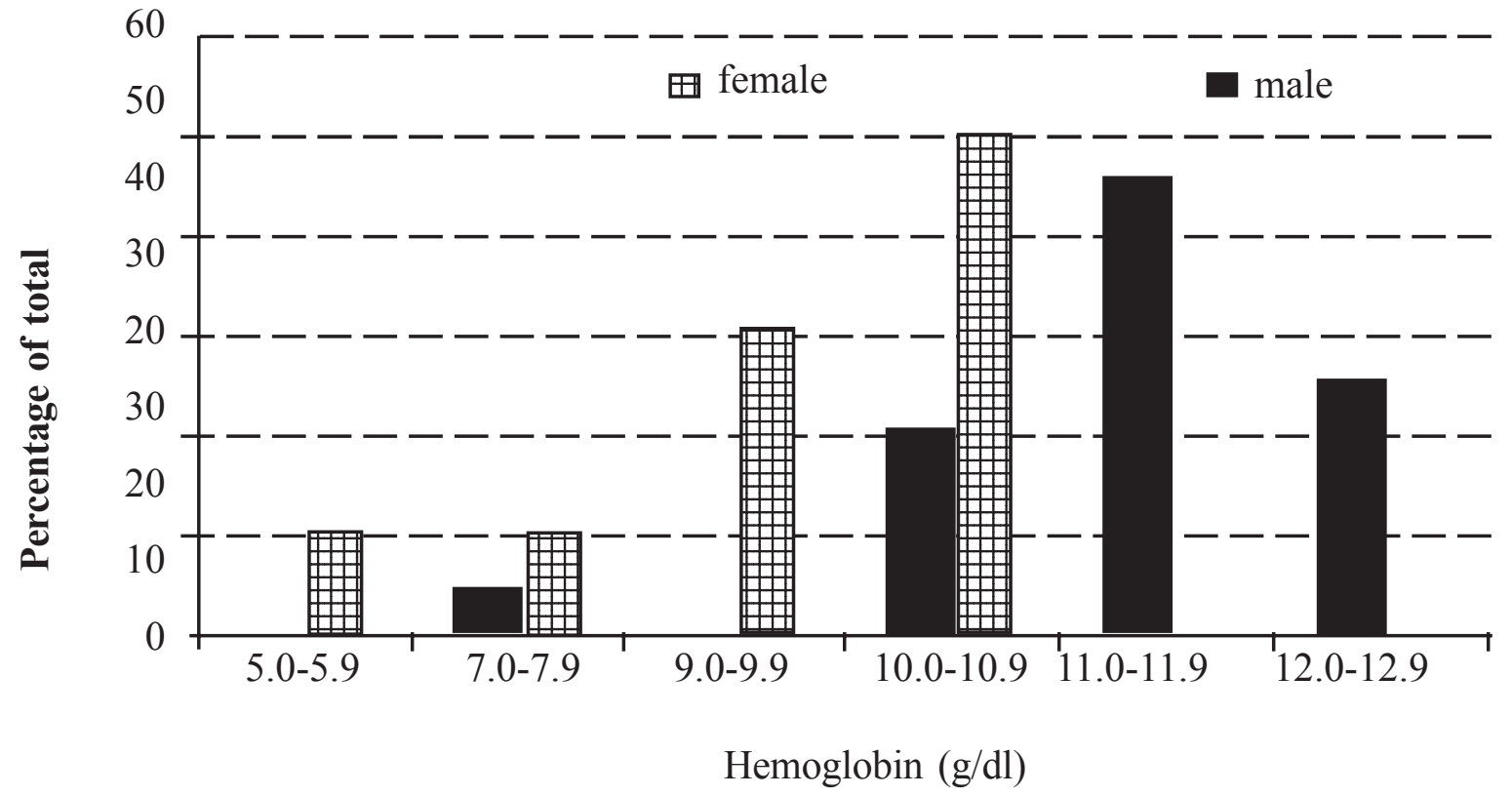

Figure 1: Hemoglobin level in male and female IDA cases. 


\section{Conclusion}

The finding of our study signifies the increased lipid peroxidation and decreased antioxidant status in iron deficiency anemia both in male and female cases. Our study was limited to few patients, the vitamins and iron supplementation was not performed. Further research is recommended to identify the specific risk factors for IDA. Further studies on lipid peroxidation and antioxidant status after vitamin and iron supplementation is being carried out in the laboratory.

\section{References}

1. G.H. Guyatt, A.D. Oxman, M. Ali, et al. Laboratory diagnosis of iron deficiency anemia: an overview. J Gen Intern Med 1992; 7: 145-53.

2. J.M.T. Willoughby, S.M. Laitner. Audit of the investigation of iron deficiency anemia in a district general hospital. Postgrad Med J 2000; 76: 21822.

3. K.V. Patel. Epidemiology of anemia in older adults. Semin Hematol 2008; 45(4): 210-17.

4. K. Tolentino, J.F. Friedman. An update on anemia in less developed countries. Am J Trop Med Hyg 2007; 77(1): 44-51.

5. P.M. Krishnamoorthy, P.R. Natesh, D.M. Mohan, et al. Role of oxidative stress and antioxidants in children with IDA. Int $J$ Collaborative Research on Internal Medicine and Public health 2010; 2(1): 2-18.

6. P.M. Clarkson, H.S. Thompson. Antioxidants: what role do they play in physical activity and health? Am J Clin Nutr 2007; 72: 637S-46S.

7. K. Rahman. Studies on free radicals, antioxidants, and co-factors. Clinical Interventions in Aging 2007; 2(2): 219-36.
8. D. Fusco, G. Colloca, M.R.L. Monaco, et al. Effects of antioxidant supplementation on the aging process. Clinical Interventions in Aging 2007; 2(3): $377-87$.

9. L.A. Pham-Huy, H. He, C. Pham-Huy. Free radicals, antioxidants in disease and health. Int J Biomed Sci 2008; 4(2): 89-96.

10. J. Imlay. Pathways of oxidative damage. Annu Rev Microbiol 2003; 57: 395-418.

11. S.P. Sanghani, V.A. Haldankar, K.K. Shalia et al. Comparative analysis of RBC membrane lipids in thalassemia and iron deficiency anemia in relation to hypochromic and oxidant injury. Indian J Clinical Biochem 2001; 16(1): 116-21.

12. W. Luman, K.L. Ng. Audit of investigations in patients with iron deficiency anemia. Singapore Med J 2003; 44(10): 504-10.

13. S. Shams, H. Asheri, A. Kianmehr, et al. The prevalence of iron deficiency anemia in female medical students in Tehran. Singapore Med J 2010; 51(2): 116-9.

14. J.A. Buege, S.D. Aust. Microsomal lipid peroxidation. Methods Enzymol 1978; 52: 302-10.

15. Z.Y. Jiang, J.V. Hunt, S.P. Wolff. Ferrous ion oxidation in the presence of xylenol orange for detection of lipid hydroperoxides in low density lipoprotein. Anal Biochem 1992; 202: 384-9.

16. P. Kakkar, B. Das, P.N. Viswanathan. A modified spectrophotometric assay of superoxide dismutase. Ind J Biochem Biophys 1984; 21: 130-2.

17. K.A. Sinha. Colorimetric assay of catalase. Anal Biochem 1972; 47: 389-94.

18. J.J. Rotruck, A.L. Pope, H.E. Ganther, et al. Selenium: Biochemical rates as a component of glutathione peroxidase. Science 1973; 179: 588-90.

19. E. Beutler, O. Durgun, B.M. Kelly. Improvement method for the determination of blood glutathione. J Lab Clin Med 1963; 51: 882-8. 
N. L. Madhikarmi et al, Effect of iron deficiency anemia on lipid peroxidation and antioxidant systems

20. S. Natelson. Estimation of Vitamin A, Vitamin C and alpha-tocopherol. In: Charles C, Thomas WB. Techniques of Clinical Chemistry. $3^{\text {rd }}$ ed, USA: Illionois; 1971: 162-758.

21. H. Baker, O. Frank, De B. Angelis, et al. Plasma tocopherol in man at various times after ingesting free or acetylated tocopherol. Nutr Rep Int 1980; 21: $531-6$

22. I.F. Benzie, J.J. Strain. The ferric reducing ability of plasma (FRAP) as a measure of "antioxidant power": the FRAP assay. Anal Biochem 1996; 239 : 70-6.

23. S.M. King, C.M. Donangelo, M.D. Knutson, et al. Daily supplementation with iron increases lipid peroxidation in young women with low iron stores. Exp Biol Med; 2008: 233: 701-7.

24. S.M. Hattangadi, H.F. Lodish. Regulation of erythrocyte life span: do reactive species set the clock? J Clin Invest 2004; 117: 2075-77.

25. H. Ye, T.A. Rouault. Human iron-sulfur cluster assembly, cellular iron homeostasis and disease. Biochemistry 2010; 49(24): 4945-56.

26. Kurata M, Suzuki M, Agar N S. Antioxidant systems and erythrocyte life-span in mammals. Comp Biochem. Physiol. B. 1993; 106: 477-7.

27. V. Gadjeva, D. Kuchukova, R. Georgieva. Vitamin combinations reduce oxidative stress and improve antioxidant status in patients with iron deficiency anemia. Comp Clin Path 2005; 14: 99-104.

28. B. Halliwell, S. Chirico. Lipid peroxidation: its mechanism, measurement, and significance. Am J Clin Nutr 1993; 57: 715S-725S.

29. A. Garg, P. Abrol, A.D. Tewari, et al. Effect of vitamin A supplementation on hematopoiesis in children with anemia. Indian J Clinical Biochem 2005; 20(1): 85-6.

30. T.P.A. Devasagayam, J.C. Tilak, K.K. Boloor, et al. Free radicals and antioxidants in human health: current status and future prospects. JAPI 2004; 52: 794-803.

31. R.C. Rose, A.M. Bode. Biology of free radical scavengers: an evaluation of ascorbate. FASEB $J$. 1993;7: 1135-42.

32. L.J. Machlin, A. Bendich. Free radical tissue damage: protective role of antioxidant nutrients. FASEB J. 1987;1: 441-5.

33. Ji LL. Antioxidants and oxidative stress in exercise. PSEBM. 1999; 222: 283-92.

34. E. Casanueva, F.E. Viteri. Iron and oxidative stress in pregnancy. J Nutr. 2003;133: 1700S-08S.

35. K.P. Ashok, G. Rajagopal. Lipid peroxidation in erythrocytes of patients with type 2 diabetes mellitus. Indian J Clinical Biochem. 2003; 18(1): $71-4$. 